\title{
"MumCare": An Artificial Intelligence Based Assistant
}

\author{
K.W.M.C.Maduwantha ${ }^{1}$, V.N.Vithana ${ }^{2}$ \\ ${ }^{1}$ Department of IT, SLIIT Academy Pvt Ltd, Panadura, Sri Lanka, \\ Email: kwmcmaduwantha@ieee.org \\ ${ }^{2}$ Department of IT, SLIIT Academy Pvt Ltd, Colombo 03, Sri Lanka, \\ Email: nipunika.v@sliit.lk
}

\begin{abstract}
Bringing a new life to the world is a wonder to every mother. Experience faced by pregnant mothers vary from one pregnancy to another pregnancy. There is a vast amount of information available on the Internet and printed materials. Yet, this knowledge is too complex or lengthy and very few applications provide customized information to pregnant mothers. In a time where smart phones have become a necessity in our life, a mobile app is one of the easiest ways to obtain prenatal information. Hence, we have developed a mobile application to help pregnant mothers. This application includes an artificial intelligence (AI) based chatbot. AI chat bot communicates and guides the mother in a way that creates the illusion as if they are talking to their unborn child. The Spiral Model was used as the development methodology and the application was developed in an environment of continuous integration and deployment with GIT personal repository. This application was implemented using React Native and the Node.js. Chat bot was created with Dialogflow agent and integrated with the firebase through the Google cloud functions. Some existing applications were studied to identify the features and limitations of current pregnancy care mobile applications. This solution is realistic and successful and it has an upgradable model of growth. The rapid development of the Internet and mobile devices in the world has changed people's lifestyles. This mobile app will be helpful for pregnant mothers living in rural as well as in metropolitan areas alike and can enhance education and health.
\end{abstract}

Keywords: Pregnancy, Artificial Intelligence, Chatbot, Natural Language Processing, Mobile Health, Maternity Records.

\section{INTRODUCTION}

It can be very challenging for mothers to be pregnant particularly when they are experiencing it for the first time. Most mothers rely on information from known friends and relatives. Conditions and problems faced by pregnant mothers vary from one pregnancy to another. Thus, this information may not be accurate or relevant at all times. This could make the problems worse and lead pregnant mothers to an uncomfortable condition. [1].

As smartphones have become more and more popular today, pregnant mothers can use them as a way to monitor their pregnancy through computerized maternity records. This would be a simple and convenient way to access knowledge about pregnancy because mHealth is the innovative use of new electronic technology to provide and improve health care services. The computerized pregnancy reports consist of eating schedules, routines and all the things to be performed regularly by pregnant women before their due date. Therefore, mobile pregnancy applications have become a popular way to easily get pregnant at the push of a button at a very low cost. Even though there are many such applications, very few of them provide customised features to pregnant mothers.

Providing a customised service which cater individual needs pf pregnant mothers can help reduce pregnancy related depression and distress. A distinct advantage of mobile application is that it is available anytime and everywhere around the clock. Thus, this system intends to allow pregnant mothers to be aware of changes during pregnancy and take the appropriate steps to prevent unintended issues.

\section{A. Aim and Objectives}

The main aim of this project was to develop an artificial intelligence-based assistant to guide pregnant mothers through the childbearing.

The Objectives were:

- To identify and evaluate existing artificial intelligence based chatbot systems and pregnancy care applications.

- To design and develop artificial intelligence-based chatbot suitable for pregnancy care application.

- To design and develop more user-friendly pregnancy care application.

- To deploy the pregnancy care application which includes artificial intelligence-based chatbot.

\section{B. Project Realisation}

The motivation to do this project was the developer wanted to bring something valuable to society and help solve problems of pregnant mothers.

Then the idea was to create a mobile application including the chatbot to guide mothers through the course of pregnancy. It has been discovered that none of the existing applications has artificial intelligence-based chatbot which provides useful information. The MumCare App provide information to pregnant mothers in a very user-friendly manner and chatbot chat with the mother as the unborn child. This is a novel idea to bring knowledge and advice to pregnant mothers.

Although the chatbot is the distinctive aspect of this application, it was not only the chatbot itself that centred the development. Other than that, daily reminders and pop-up notifications, emergency dialling feature, kick counter, 
pregnancy tips are other features available in the MumCare App. This mobile application also supports pregnant mothers to engage in small activities such as games. This is beneficial during their pregnancy to improve maternal mental wellbeing. Therefore, this application provides various benefits including virtual chat bot, user friendly interfaces, great performance and access to health-related information from around the world.

\section{LITERATURE REVIEW}

This section explores the major research topics related to this project and the researcher has summarised previous researches on Artificial Intelligence, Artificial Intelligencebased Chatbots, Mobile Health (mHealth) and Mobile Phone-Based Pregnancy Support Systems.

\section{A. Artificial Intelligence}

Human toyed with the idea in the early centuries that computers could achieve the same stages of thought as the human brain. The robots' thoughts and renderings can be traced back to the Greek myths and Chinese and Egyptian history. Flasinski's "Introduction to Artificial Intelligence" book shows that since ancient times, certain basic philosophical questions in artificial intelligence have been of considerable significance in philosophy, and thinkers such as Aristotle, St. Thomas Aquinas, William of Ockham, etc., have asked certain questions that could not be answered until the first computers were invented in the twentieth century. Allen Newell and Harbert A. Simon were designed, the first artificial intelligence system which named as 'Logic Theorist' at the Carnegie Mellon University in 1955 [2].

The world has been making several technical strides in AI since the first AI system was built and now it brings to mind robots that can behave like a human [3]. These are more complex and complicated structures that have been designed to tackle situations where problem solving without human intervention could be needed. There were two parts in the artificial intelligence: weak artificial intelligence and strong artificial intelligence [4]. Weak artificial intelligence includes a machine programmed to execute a specific purpose, and strong artificial intelligence-based systems execute the tasks encountered as a human being [4].

Artificial intelligence has drawn interest in developing countries as a key to development, and is growing rapidly. Artificial intelligence is rapidly changing as technology progresses to support several diverse sectors. The technologies can be extended to numerous fields; thus, the artificial intelligence implementations are infinite.

1) Natural Language Processing: Natural language processing, commonly simplified as NLP, is a branch of artificial intelligence that uses the natural language to deal with the contact between machines and humans. NLP 's overall goal is to learn, translate, understand, and make sense of the human languages in a meaningful way. Most NLP strategies are based on learning the computer to infer meaning from human languages [5]. NLP will support you with a lot of activities and the areas of use appear to only grow every day. In the healthcare sector NLP is especially booming. This technology increases the quality of treatment, the diagnosis of illness and lowers costs as healthcare institutions are rapidly embracing electronic health records. The idea that clinical reporting can be enhanced implies more comprehension and helping people by quality treatment.

A difficult challenge is designing a technology that knows the natural language. There are large a number of natural languages, they contain infinitely many sentences. There's still a lot of doubt about the natural language [6]. Also, many words have several meanings and several times the same phrases have different meanings in various ways. Because of this it is a difficult challenge to develop applications that comprehend a natural language. Importance of NLP is due to the fact that there is a massive volume of data in the world wide web, at least 20 billion pages, that can be used as a major resource, as long as useful information can be retrieved by NLP from this [5].

\section{B. Chatbot systems}

As a human-computer interface, Chatbots have played a major role in the last few years. A chatterbot or chatbot is a computer program that mimics human conversation spoken and written. Chatbots are currently leading the market and bringing it into the era of Artificial Intelligence. The chatbots do human jobs, practice it and master human strategies as well [6]. Chatbots conduct conversations with people, and developers typically hope that users will not realize they're talking to a robot [7]

Generally, the chatbot was written in three modules: the user interface, an interpreter, and a knowledge base [8] There are two types of chatbots: one whose functions are based on a set of rules and another one which uses artificial intelligence and this is the more advanced version. When artificial intelligence used in a chatbot, the chatbot always gets smarter as it can learn new things from the conversations with the human. Doshi, S. V., Pawar, S. B., Shelar, A. G. \& Kulkarni, S. S (2017) define that as a program, chatbots are an attempt to simulate typed conversations and they have been deployed on the internet for the educational, guidance, customer service, and entertainment purposes.

The first chatbot in the world is ELIZA which works on the pattern matching system developed by the German scientist, Joseph Weizenbaum in 1966 [9], [7]. Human users could chat with ELIZA and this chatbot was initially created to respond as a psychotherapist, and users can have a meaning full conversation [7]. Since ELIZA was created by J. Weinbaum, the world has been headway in the development of intelligent chatbots. As a result, in 1972, PARRY was created by Kenneth Colby, Richard Wallace created ALICE which was considerably complex in 1995, and there was a range of new chatbot architectures have been deployed [10].

ALICE was a rule-based chatbot based on Artificial Intelligence Mark-up Language (AILM). The AILM based chatbots are easy to implement, those are lightweight and efficient to handle and AILM still uses today [11]. Jack's research shows [9], an Artificial Intelligence Mark-up Language is obtained from Extensible Mark-up Language (XML) which is used to build conversational virtual assistance and it has more than 40,000 categories, where each one has a combination of different patterns and its response. His paper gives detailed information about the architecture, design and development of chatbots.

Empowered by artificial intelligence, chatbots are rising as new technologies in business potential. In the market 
place, customers who used artificial intelligence-based applications were also reacting positively to chatbots as they use chatbots for sales, guidance, customer service, entertainment, health care, education, and more. The Marketing Science [12] emphasise 'The market size of chatbots is expanding quickly, from \$250 million in 2017 to more than $\$ 1.34$ billion in 2024' and the use of this artificial intelligence-based applications span across individual services to commercial purposes. In the year 2020 , over $85 \%$ of customer interactions will be handled by bots. Therefore, in the future customer supports jobs seems to replace by the chatbot and also, people find that have a chat conversation with a chatbot is well-being rather than making a phone call. However, the use of artificial intelligence-based chatbots is in its childhood, but the future is exciting.

In MumCare mobile application artificial intelligencebased chatbot technology is used to help pregnant mothers in a way that creates the illusion as if they were talking to their unborn child where this will help stabilise their mental as well as physical health.

\section{Mobile Phone-Based Pregnancy Care}

Being pregnant can be very challenging for mothers especially when they are first expecting it. Most depend on knowledge from one pregnancy to another that may not be reliable as experience from each pregnancy can vary from one pregnancy to another, and from one person to another. Mobile phone-based pregnancy support will position itself as a significant breakthrough method for educating women about pregnancy, tracking their own and child's growth, tracking medical check-ups, and stabilise their mental as well as physical health [1].

The current trend in the healthcare sector is to connect patients, doctor and hospitals to provide all patient with optimum treatment. Patients also say their physicians should be open to tacking a part in video conversations [13]. Mobile pregnancy applications have become a modern way of providing readily accessible maternity contents, simply by pressing a button, at little or no cost [14]. However, the main concern is over the quality of these developed applications, thus the accuracy of information received by mobile pregnancy applications needs to be tracked [15].

The provision of strong mobile network coverage and mobile phone technology will help minimize the stress and anxiety related to pregnancy and its complications through a support system that is available anytime and anywhere. The system should allow the pregnant women to be aware of changes during pregnancy and take the necessary actions to avoid unexpected changes.

\section{D.Similar Applications}

Pregnancy week by week by Amila [16], Pregnancy Tracker by Mobile Dimension LLC [17] and Pregnancy Tracker by What to Expect [18] is the highly-rated pregnancy care applications in Google play store and Apple Store. These applications have been used to understand the capabilities that mobile applications for pregnancy currently provide and those that they are lacking. These applications are very helpful and useful for the weekly tracking of their pregnancy and they have over one million downloads with average ratings of 4.7 to 4.9 .

Get pregnancy tips, week by week pregnancy tracking, calculate due date, pregnancy calendar, weight tracking, kick counter and pregnancy diary can be considered as a common functionality in these three applications. However, none of these applications was used chat-bot. Therefore, chatbot is the major difference between existing applications and the MumCare. In addition, the application includes an emergency dialling system and it supports women by computerized maternal recoveries during their pregnancy.

\section{E. Conclusion}

This analysis reassure the reader that it is important for the mHealth and artificial intelligence assistants (chatbots) to use it to improve self-care for pregnant women. During this phase, it was discovered that the artefact created has a unique feature and with some additional features and added functionality. It will guide pregnant mothers in a way that creates the feeling they talk to their unborn child and help stabilize their physical and mental health.

\section{DESIGN AND IMPLEMENTATION}

\section{A. Planning}

This application was implemented using Spiral methodology that will certainly yield a release of the version by the potential implementation. Every spiral can be named as a loop in the spiral model and each loop is a different method of creation and there are four phases: Planning, Risk Analysis, Engineering and Evaluation. The tasks started with an initial process of preparation and collecting requirements to prepare the project proposal, the appropriate resources and the Gantt chart to assign and allocate the time and resources available. The engineering process took places with the application being tested, coded and deployed, using continuous integration and continuous deployment.

In addition, the version-based feature releases involved defining the threats in the project along with the supervisor and mitigating the post-release problems. With development, testing, and continuous integration and deployment will take place after the task engineering section within the version was developed. This creates a platform for effective pre and post-version review and assessment. Any bugs detected will be moved for fixing in the next release of the version.

\section{B. Architecture}

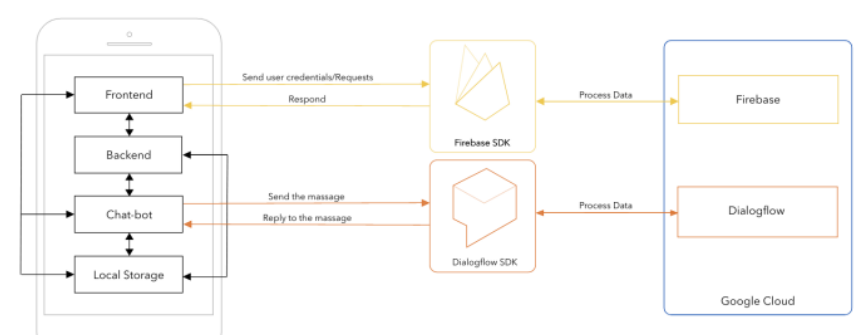

Fig. 1. Architecture diagram which illustrates interconnectivity of the application.

Above architecture diagram (Fig.1) outline the description and interconnectivity of this application and also describe how the chatbot communicates with users and how it transfers and receives data from local storage and cloud storage. There are two separate data stores for the MumCare; the Firebase Realtime Data Base handles user details and events, whereas the Local Storage used to store user profile picture. The Dialogflow and Firebase are connected together through the google cloud to share the 
data when the chatbot needs to retrieve user information. As in the mentioned in Fig 1, when user completed registration process their user credential will sent to the firebase authentication because it provides backend services and SDKs to authenticate user with the MumCare application. When user login to the application the user name and password will be sent to the firebase and validate to find whether the user has already registered.

The Firebase and the Dialogflow were connected through the Google Cloud Platform and the database of the MumCare should be registered in the Dialogflow in order to communicate among them. When users have conversations with the chatbot, the messages will be sent to the Dialogflow agent and for any data requests, the agent could access to the database and retrieve the necessary data and then send them to the backend of the application.

\section{Implementation}

MumCare offers numerous options such as kick counter, baby name list and reminder to make it more user friendly. These are the features of the MumCare mobile application.

- Baby Chatbot

- Kick Counter

- Pregnancy Calendar

- Reminder

- Baby Name List

- Mind Relaxing Game (Tic Tac Toe)

- Computerised Maternity Records

- Emergency Dialling System

1) User Account Structure: Every MumCare user needs to have an account that stores the data directly in local storage. React Native carries certain efficiencies, and when creating an authentication and handling user functions can often be difficult to deal with. Thus, MumCare was using Firebase Authentication to handle user accounts and authentication. Firebase Authentication tightly integrates with other Firebase applications, using industry standards such as OAuth 2.0 and OpenID Connect, making it easier to incorporate with any backend.

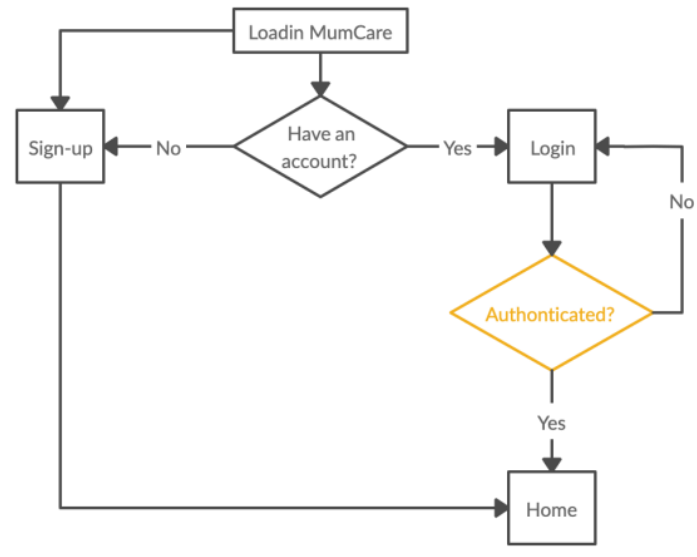

Fig.2. User authentication flow interact with Firebase

Firebase Authenticate helps users to authenticate with their emails and passwords, it also manages resetting emails and passwords. The user has to provide their credentials to sign up to the system and these credentials will be the email address and password for the account. Then, the Firebase
Authentication SDK sends those credentials to the Firebase (Fig.2).

2) Pregnancy Calendar: The pregnancy calendar is a detailed guide which illustrates the baby's growth with all the changes taking place and each week of pregnancy includes a description of the baby's development. The application would have an attractive user interface design to demonstrate baby's growth weekly with current week, day, how many days remained, the trimester, baby's measurements and photos.

Moment.js was used to parse, validate, manipulate, and display dates and times in pregnancy calendar. This can be installed with a simple node command "npm install moment --save". Using this library, the current week and day of the pregnancy will be calculated, and identified number of days left to delivery. Also, Moment.js is used to illustrate baby's development by images based on the current week. Depending on the due date which is user entered when registering to the application the system will be customised.

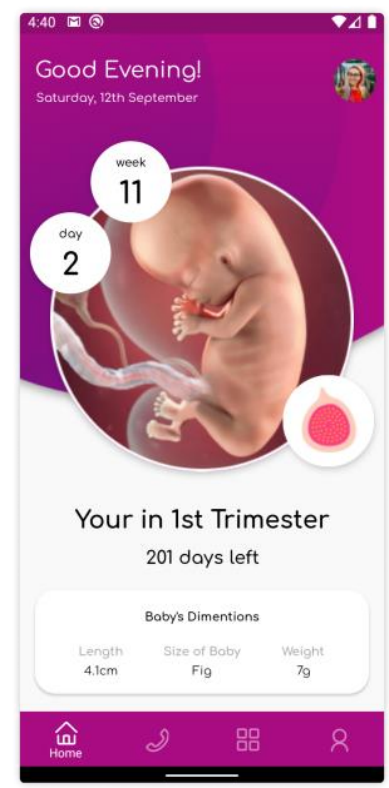

Fig. 3. Pregnancy calendar in the home page of the application.

3) Mind Relaxing Activities (Tic Tac Toe): Not all games for kids, sitting down with a board game will really get your mind off the pregnancy stresses. They are fun and take you back to the good old days of childhood when it all was much simpler. Similarly, board games are a fun way to connect and spend time with your other half, rather than pacing in front of a TV without talking. Board games initiate conversation, and while they are vintage and a little classic, they are more relaxing than video games that can put strain on eyes.

Therefore, MumCare will provide Tic-Tac-Toe game which is a virtual Board/Strategy game using "tictactoeagen" node module to determine the system's next move. This can be installed with simple node command "npm install tictactoe-agent --save". When click on the start game it will start to render game component. The board divided in to 9 tiles ( $3 \times 3$ grid). To play the game, the user must start by entering a circle, after the choice has been submitted, the tictactoe-agent will identify the index of the tile (index 0 ) which the user has selected. Then, the tictactoe-agent will reference the victory conditions $([0,1,2] /[0,3,6] /[$ 
$0,4,8]$ ) as shown in the array shown below Fig.4 and it will select index 04 as a suitable index which has most winning chances to the system, and which will reduce the users winning chances (Step 04). Then that index will be represented by a cross. The user will then enter a circle into index 3 , at which point, the previous steps will be repeated until the game is won or drawn (as shown from steps 5 to 9).
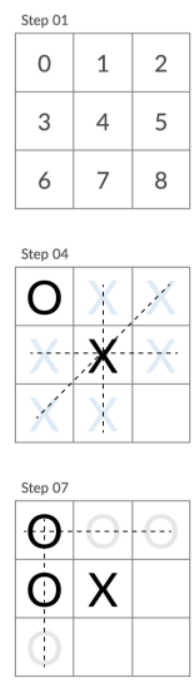
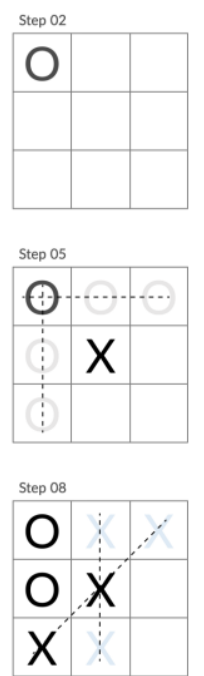
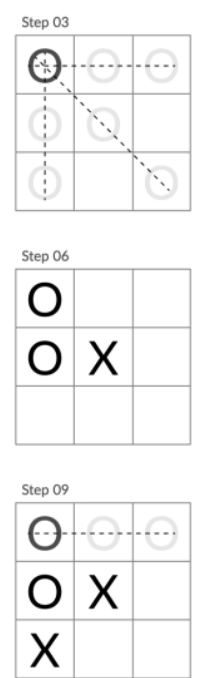

Fg. 4. How tic-tac-toe game works (example)

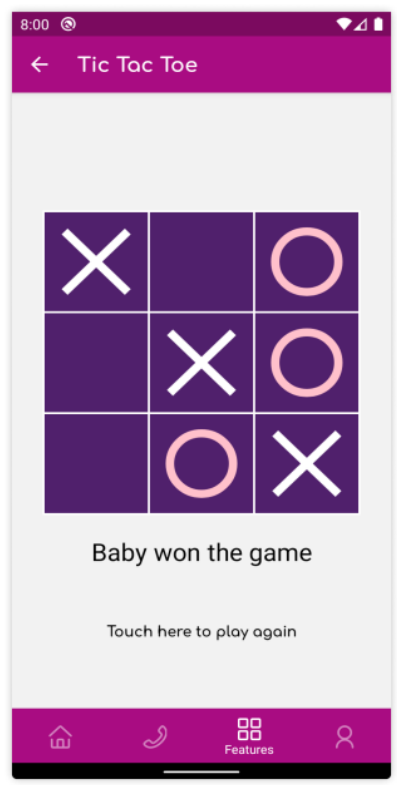

Fig. 5. Tic-Tac-Toe game page in the application.

4) Chatbot: This is the unique component of this project, Artificial Intelligence-based chatbot. The Dialogflow is used to develop the chatbot which gives users to interact with their product by developing text and voice-based conversational interfaces such as chatbots and voice applications powered by NLP. Dialogflow is a natural language processing-based platform that makes it easy to design and integrate a conversational user interface into mobile applications, web applications etc. As mentioned in section 3.5.4, the front-end of the chatbot has been implemented in the application and the back-end was implemented in the Dialogflow. When the fulfilment for an intent was enabled, Dialogflow responds to that intent by calling a service that has been defined by the developer. There is an in-built code editor for Dialogflow called 'inline editor' which can be used to crate fulfilment code and deploy it to Cloud Functions.

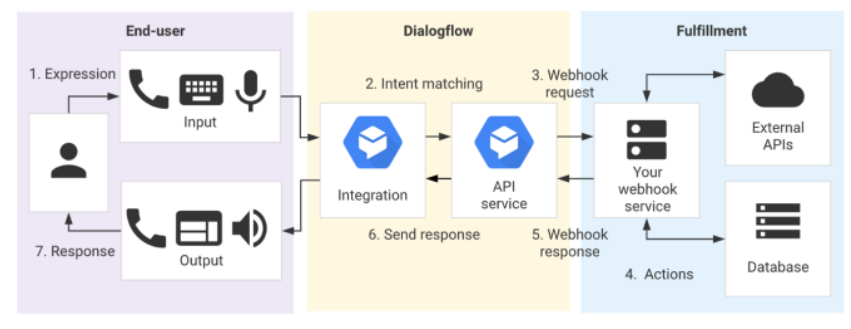

Fig. 6. Processing flow for fulfilment in Dialogflow

Above Fig.6 illustrates the processing flow for fulfilment. When the user enters their request, the Dialogflow matches the requirement with an intent to extract parameters. It will send a webhook request to the webhook service and this service message consists of the action, parameters and the response. The actions can be API calls or data retrieves from the database. Finally, as in step 5, the webhook response will be sent to the Dialogflow and the Dialogflow sends the response to the end-user.

The Dialogflow service for application can be installed in React Native environment with a simple node command "npm install --save react-native-dialogflow react-nativevoice". When the user provides the information, the user input which could also contain entities must be handled by Dialogflow. Therefore, Dialogflow has to seek the details from the web-hook to fulfil the request of the users. The data provided by the user is then sent to the web-hook along with entities so that the necessary information can be retrieved Once the Dialogflow receives the information from webhook it sends the response back to the user in the desired manner.

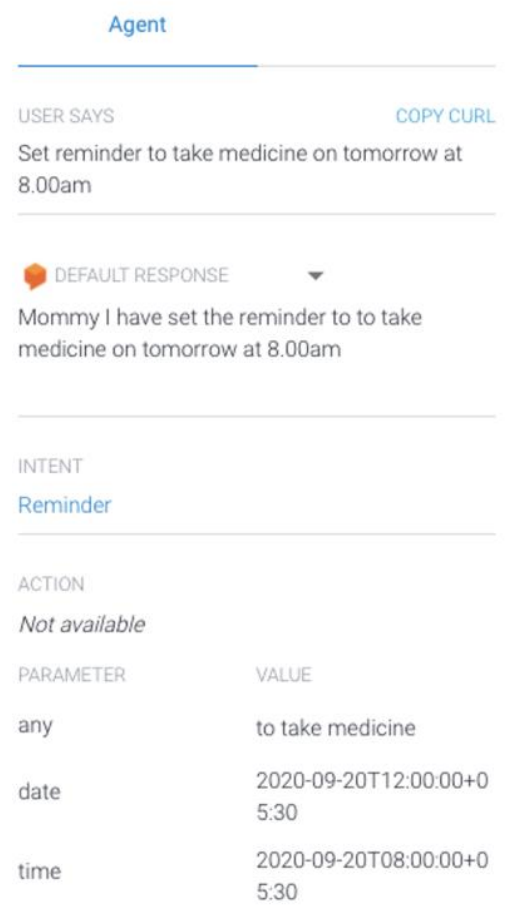

Fig. 7. Snapshot of the agent in Dialogflow consol. 
When developing the baby chatbot in application the React Native Gifted Chat was used to create front-end. There are three main functions implemented in the inline editor with three intents to make reminders through the chatbot, to get existing reminder details and get pregnancy information by week.

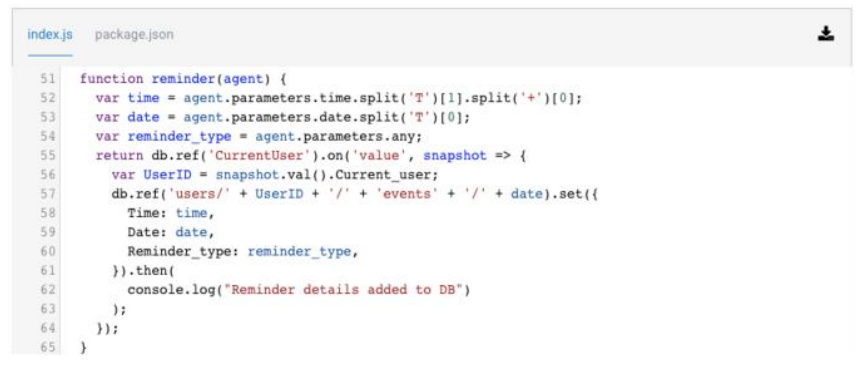

Fig. 8. Code snippet of the 'reminder' function

The Fig. 8 illustrates one of those three functions which were implemented by the developer. The users can make reminders through the application as well as the chatbot without using the calendar feature. The function will read the reminder details from the agent by providing a simple code segment: 'parameters.entity_name'. The 'time', 'date' and 'any' are the three entities used in this intent. To store the values in the database current users' ID should be identified first because there could be many users and each user has a separate database. Then the user ID will be initialised to the variable called 'UserID' and the data should set the database using the user ID as a reference (line 57 in the Fig.8).

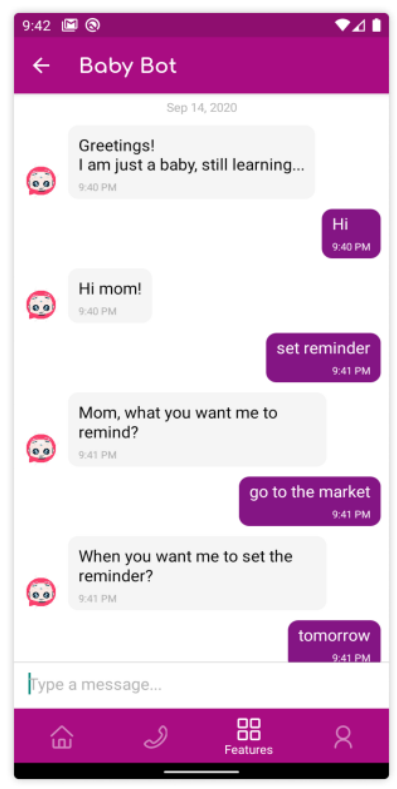

Fig. 9. Sample chat preview of the baby chatbot.

\section{RESULTS AND DISCUSSION}

In this research, the researcher simulated multiple scenarios to check the performance and functionalities. The scenarios were divided into main 4 stages. One of the scenarios was used to check the Pregnancy Calendar by inserting different due dates and the second scenario was used to check the performance and reliability on chatbot by asking different questions and requirements. Then the third scenario was inclusive to test the emergency dialling system, accuracy of the tic-tac-toe game and accuracy of the reminder function. Finally, the last scenario has included testing the whole system, flow and integration of the all components. During the 4 simulation phases, all the scenarios turned out to deliver a good performance. Nevertheless, the researcher has found that the results in some areas need to be improved and in other areas, the results were more than expected.

The agent's average response time is less than 5 seconds (time period from 2020/08/19 to 2020/09/19). The response time of the agent depends on the user's request. For an example, for a small talk conversation, the agents' response time averages from 1-3 seconds and if the agent gathers data from the database, the average response time increases to 3 5 seconds and in some cases, more than 5 seconds. When evaluating the Firebase Performance test results, the average application start time is less 3-6 seconds.

When testing the reminder function with Firebase Cloud Messaging (FCM), pop-up notifications cannot be received if the device is offline. To overcome this problem an in-built function using react native was developed to send push notifications. This allows all notifications and reminders from the application to be received when the device is offline.

When creating the Chatbot the researcher faced some difficulties to get data from the database and since the time has been limited some of the functionalities could not be implemented. As the agent is hosted on the web, further development can be done in the future through Dialogflow.

Finally, after conducting experiments on the application many functionalities proved to be working as expected. To develop this system into a commercial product using the latest technologies such as artificial intelligence-based chatbot that uses a large database and more technical improvements are required. Further encouragements for additional improvements will be mentioned later in the report to create a better application based on the evaluation of existing systems and evaluation results.

\section{EVALUATION}

Three methods were used to evaluate the artefact to see whether it is fit for the purpose of the system. First, the artefact was analysed at the start of the project and afterwards modifications were made according to personal preferences. Finally, the end users of the application were allowed to use the application and provide feedback to be assessed and evaluated. After combining the above techniques, MumCare can be considered as a successful project.

To evaluate the potential for the artefact, a group of 6 expecting mothers were gathered and asked to try the MumCare mobile application. After having a chance to try out the application they were asked to complete a survey about their opinions about the MumCare, what they liked and disliked about it, the drawbacks and then the features they willing to have in the future. 
MumCare

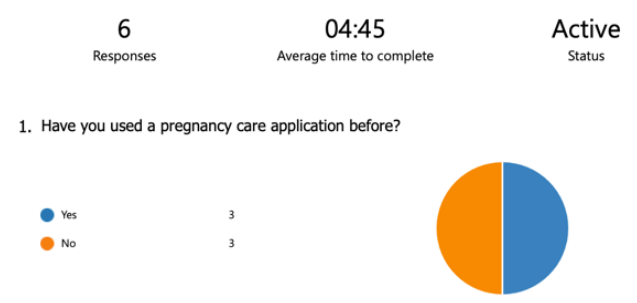

2. Do you think an application like the one used could be useful during pregnancy?

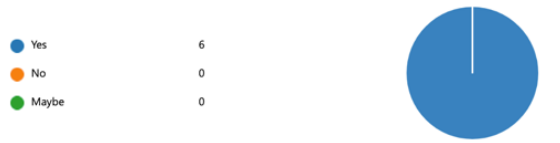

3. Why?

6

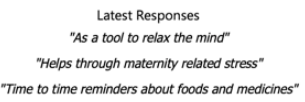

Fig. 10. 'MumCare' Evaluation Results - Part A

4. What additional features could be added to make it a better application?

6

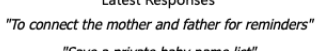

"Save a private baby name list" "Daily water reminder and tracker"

5. Additional Comments?

6

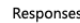

6. What are the most favourite features in MumCare?

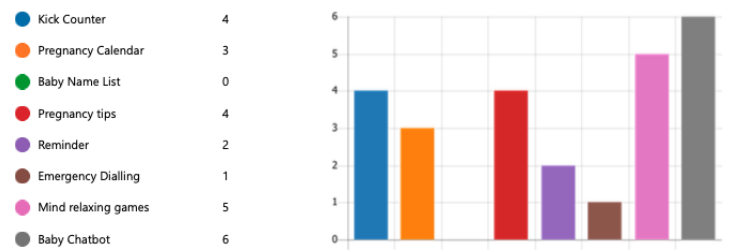

7. Would you recommend MumCare ?

$$
\text { - yes }
$$$$
\text { No }
$$

Fig. 11. 'MumCare' Evaluation Results - Part B

\section{FUTURE WORK}

It would be tedious and needs a lot of experience to get the whole project to a stage with certain implementations that already exist. The focus will be based solely on the artificial intelligence-based chatbot, since this idea will be built independently and within a limited period of time. Hopefully, a couple of features can be added during the allocated time period with successful time management, but a completed product can be expected in future. Interactive 3D models will illustrate the development of the baby and track the mother's walking patterns by using the smart devices' built-in sensors which will provide useful alerts.

To increase the usefulness of the MumCare, additional features to interact with the current notification system could be added. The notification system should be developed further to send notifications to both mother and father. The weight tracking system to be developed as further implementation and this will help to keep track of mother's weight. At the outset and regularly during pregnancy, track your pregnancy weight gain and compare your progress with suggested ranges of safe weight gain.

\section{CONCLUSIONS}

During the research the developer identified the possible needs for a new technique of guiding pregnant mothers through an artificial intelligence-based assistant, using both end-user surveys and analysing the existing applications. In this thesis, various functionalities of the pregnancy care application were researched and it was found that none of the existing applications have a chatbot.

Due to the unavailability of a pregnancy care application with an artificial intelligence based chatbot, MumCare demonstrates a working proof of concept to potential users and opens doors for further development to allow more features and functionalities. Furthermore, during the evaluation phase, the feedback from short survey given to expecting mothers after allowing them to gain first-hand experience of using the application has brought mainly positive feedback and other suggestions with good comments.

\section{ACKNOWLEDGMENT}

I would also like to thank Mr. N. Kalansooriya, Full-stack developer at Leafycode (Pvt) Ltd., Sri Lanka, for his valuable contribution in guiding me in using React Native successfully.

Finally, I would like to express my appreciation for my friends at the SLIIT Academy because the concept for this project would not have evolved without the invaluable contribution of them.

\section{REFERENCES}

[1] S. M. Tabatabaei, R. Ghaedi, E. A. Khonsaraki and A. Talebi, "Application of mobile health to improve self-care among pregnant women: A literature review," Journal of Medical Physiology, vol. 5, no. 1, p. $1,2020$.

[2] M. Flasinski, Introduction to Artificial Intelligence, Springer, 2016

[3] N. Delamater, "A brief history of artificial intelligence and how it's revolutionizing customer service today," SmartMax Software Inc., 2018.

[4] H. Lu, Y. Li, M. Chen, H. Kim and S. Serikawa, "Brain intelligence: go beyond artificial intelligence," Mobile Networks and Applications, 
vol. 23, no. 2, pp. 368-375, 2018

[5] K. R. Chowdhary, Fundamentals of artificial intelligence, New Delhi: Springer (India) Private Limited, 2020.

[6] J J. Purohit, A. Bagwe, R. Mehta, O. Mangaonkar and E. George, "Natural Language Processing based Jaro-The Interviewing Chatbot," 3rd International Conference on Computing Methodologies and Communication (ICCMC), pp. 134-136, 2019.

[7] P. Nikhila, G. Jyothi, K. Mounika, C. K. K. Reddy and B. V. R. Dr. Murthy, "Chatbots using Artificial Intelligence," Intternational Journal of Research, vol. 8, no. 1, 2019.

[8] S. V. Doshi, S. B. Pawar, A. G. Shelar and S. S. Kulkarni, "Artificial intelligence Chatbot in Android system using open source programO," International Journal of Advanced Research in Computer and Communication Engineering, vol. 6, no. 4, pp. 816-821, 2017.

[9] C. Jack, "СНАТВОT: Architecture, design, \& development," University of Pennsylvania School of Engineering and Applied Science Department of Computer and Information Science, 2017.

[10] B. A. Shawar and E. Atwell, "Chatbots: are they really useful?," $L D V$ Forum, vol. 22, no. 1, pp. 29-49, 2007.

[11] M. S. Satu and M. H. Parvez, "Review of integrated applications with aiml based chatbot," International Conference on Computer and Information Engineering (ICCIE), pp. 87-90, 2015.

[12] Luo, X., Tong, S., Fang, Z. and Qu, Z., "Frontiers: Machines vs. Humans: The Impact of Artificial Intelligence Chatbot Disclosure on Customer Purchases," Marketing Science, vol. 38, no.6, pp.937, 2019.

[13] S. R. Steinhulb, E. D. Muse and E. J. Topol, "The emerging field of mobile health," Science Translational Medicine, vol. 7, no. 283, pp. $1-6,2015$.

[14] S. Emerson, C. Heavin and D. J. Power, "Workplace Health Promotion: Effects of an mHealth application on Employee Behaviour and Wellness," Proceedings of the 53rd Hawaii International Conference on System Sciences, pp. 3419-3428, 2020.

[15] A. Hussain, E. O. C. Mkpojiogu, N. Fadzil, N. Hassan and Z. F. Zaaba, "A mobile usability evaluation of a pregnancy app," Journal of Telecommunication, Electronic and Computer Engineering (JTEC), vol. 10, no. 1-11, pp. 13-18, 2018.

[16] https://amila.io/index.html. [Accessed 02 March 2020].

[17] https://www.pregnancytracker.app. [Accessed 03 March 2020].

[18] "What to Expect - The Most Trusted Pregnancy \& Parenting Brand," Available: https://www.whattoexpect.com/. [Accessed 03 March 2020]. 